\title{
IL-6 serum level and olfactory dysfunction severity in COVID-19: correspondence
}

\author{
Won Sriwijitalai $^{1}$ (i) $\cdot$ Viroj Wiwanitkit $^{2}$
}

Received: 28 January 2022 / Accepted: 31 January 2022 / Published online: 4 March 2022

(๖) The Author(s), under exclusive licence to Springer-Verlag GmbH Germany, part of Springer Nature 2022

Dear Editor, we would like to comment on the publication "Correlations between IL-6 serum level and olfactory dysfunction severity in COVID-19 patients: a preliminary study [1]." Vaira et al. concluded that "In COVID-19 patients, psychophysical olfactory scores did not show significant correlations with the plasma levels of a well-recognized negative prognostic factor such as IL-6. This observation casts some shadows on the positive prognostic value of olfactory dysfunctions. [1]." We agree that it is interesting to try to find clinical correlation between olfactory symptom and a laboratory parameter for inflammation. Regarding IL-6, it is an inflammatory marker and might be associated with many confounding factors. Nevertheless, an important concern on this report is on the statistical method. In general, a correlation analysis is based on the two continuous parameters, which are derived from measurement. In the present work, IL-6 is a continuous parameter but the psychophysical olfactory scores are derived from a scoring system and is a numerical scale from a basic counting. Therefore, the correlation should not be valid and the derived coefficient parameter cannot be further interpreted. This kind of pitfall is a common statistical error in clinical association study [2].

\section{Declarations}

Conflict of interest The authors declare that they have no conflict of interest.

\section{References}

1. Vaira LA, De Vito A, Deiana G et al (2022) Correlations between IL-6 serum level and olfactory dysfunction severity in COVID19 patients: a preliminary study. Eur Arch Otorhinolaryngol 279:811-816

2. Ludbrook $\mathbf{J}$ (2008) Statistics in biomedical laboratory and clinical science: applications, issues and pitfalls. Med Princ Pract 17(1):1-13

Publisher's Note Springer Nature remains neutral with regard to jurisdictional claims in published maps and institutional affiliations.

This comment refers to the article available online at https://doi. org/10.1007/s00405-021-06868-5.

\footnotetext{
Won Sriwijitalai

wonsriwi@gmail.com

Private Academic Consultant, Dimapur, India

2 Dr DY Patil University, Pune, India
} 